\title{
The Advanced Particle-astrophysics Telescope (APT) Project Status
}

\section{James H. Buckley ${ }^{a, *}$ on behalf of the APT Collaboration}

(a complete list of authors can be found at the end of the proceedings)

${ }^{a}$ Washington University in St. Louis,

Dept. Physics, One Brookings Drive, Saint Louis, MO, USA

E-mail: buckley@wustl.edu

We describe the development of a future gamma-ray/cosmic-ray mission called the Advanced Particle-astrophysics Telescope (APT). The instrument will combine a pair tracker and Compton telescope in a single monolithic design. By using scintillating fibers for the tracker and wavelength-shifting fibers to readout CsI detectors, the instrument will achieve an order of magnitude improvement in sensitivity compared with Fermi but with fewer readout channels, and lower complexity. By incorporating multiple Compton imaging over a very large effective area, the instrument will also achieve orders of magnitude improvement in $\mathrm{MeV}$ sensitivity compared with other proposed instruments. The mission would have a broad impact on astroparticle physics, but the primary science drivers for the mission include: (1) probing WIMP dark matter across the entire natural mass range and annihilation cross section for a thermal WIMP, (2) providing a nearly all-sky instantaneous FoV, with prompt sub-degree localization and polarization measurements for gamma-ray transients such as neutron-star mergers and (3) making measurements of rare utraheavy cosmic ray nuclei to distinguish between n-star merger and SNae r-process synthesis of the heavy elements. We will describe ongoing work including a series of accelerator beam tests, a piggy-back Antactic flight (APTlite) and the recently funded long-duration balloon mission: the Antarctic Demonstrator for APT (ADAPT).

$37^{\text {th }}$ International Cosmic Ray Conference (ICRC 2021)

July 12th - 23rd, 2021

Online - Berlin, Germany

\footnotetext{
*Presenter
} 


\section{Introduction}

APT was conceived as a probe-class mission to address two big questions: determining the nature of dark matter and understanding the physics of neutron-star mergers and their role in the origin of the heavy elements. To achieve these goals, it is necessary to improve $\gamma$-ray sensitivity from the $\mathrm{MeV}$ to $\mathrm{GeV}$ energies by at least an order of magnitude compared to existing experiments, but without a corresponding increase in mission cost. This is only possible with a new technical approach for a very large Compton and pair telescope: APT makes use of scintillating fibers read out by Silicon Photomultipliers (SiPMs) and a distributed imaging CsI calorimeter (ICC) to provide more than order-of-magnitude larger effective area and twice the field of view of the Fermi LAT.

While the APT design concept is optimized for $\gamma$ ray performance, it would be a true particle-astrophysics observatory providing complementary $\gamma$-ray and cosmicray $(\mathrm{CR})$ measurements that address the same questions: (1) The MeV-TeV $\gamma$-ray measurements would provide an order-of-magnitude improvement in DM upper limits from stacked Dwarf galaxy observations compared with Fermi. APT would be in a position to either detect DM or rule out the entire natural parameter space for a thermal WIMP. (2) The excellent sensitivity, large instantaneous field of view (FoV), and sub-degree point source localization in the $\mathrm{MeV}$ range will provide efficient alerts for multimessenger follow-ups of gravitational wave sources and short GRBs. At the same time, APT will provide measurements of the elemental abundances of rare ultra-heavy elements in the galactic CRs, providing a key method to

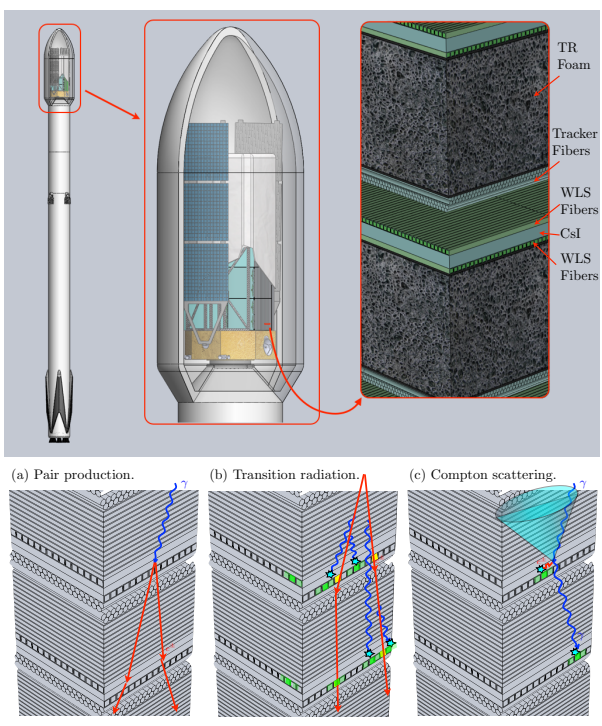

Figure 1: Top: APT in Falcon-9 faring. Bottom: APT detection modes. distinguish between a supernova or neutron-star-merger origin for r-process elements.

A first NASA APRA grant made it possible to develop and test the new technology for the mission: the imaging CsI Calorimeter (ICC) and scintillating fiber tracker. The APTlite prototype instrument flew as a piggyback on the Antarctic flight of SuperTIGER launched on Dec. 16, 2019; the 32-day flight of APTlite demonstrated the viability of the technical approach and provided a calibration of the imaging Calorimeter with CR data. The previous year, a CERN beam test demonstrated the viability of the ICC detectors for measurements of ultraheavy CR, showing no significant saturation effects in the scintillation light signal up to $\mathrm{Z}=82(\mathrm{~Pb})$ and demonstrating the large dynamic range of the ICC detectors, from single photon counting up to ionization signals from $150 \mathrm{GeV} / \mathrm{n} \mathrm{Pb}$ nuclei [1]. As the final step in this $\mathrm{R} \& \mathrm{D}$ program, we are finishing the construction of a 4-layer tracker/calorimeter instrument for accelerator testing with both a high energy electron and an inverse Compton beam. A recent NASA grant will allow us to go further and develop a full suborbital mission, the Advanced Demonstrator for APT (ADAPT), targeting a long-duration flight on a 60 million-cubic-foot balloon flight from Antarctica in the FY25 season. This instrument will integrate all of the major components of the APT mission and will provide some unique measurements of GRBs or other transients over a broad range of energies from $<1 \mathrm{MeV}$ to $\mathrm{GeV}$ that surpass the capabilities of other existing experiments.

At this ICRC, a number of papers discuss aspects of the APT project including: detailed 
simulation studies of APT [2], results of the CERN beam test and APTlite flight [1], and development of real-time computational methods for in-flight GRB reconstruction [3]. This paper focuses on overview of the continued research and development for APT and provides a detailed description of the ADAPT and APT instruments and detector subsystems.

Analysis of Fermi LAT data on Milky-Way satellite dwarf galaxies has resulted in some of the most powerful constraints on models of WIMP dark matter up to $>100 \mathrm{GeV}$ masses [4] and underscores the important role of indirect detection on solving the dark matter problem. With more than an order of magnitude improvement in exposure factor in the $\mathrm{GeV}$ range and with extended coverage of the continuum spectrum down to $\mathrm{MeV}$ energies, APT would be

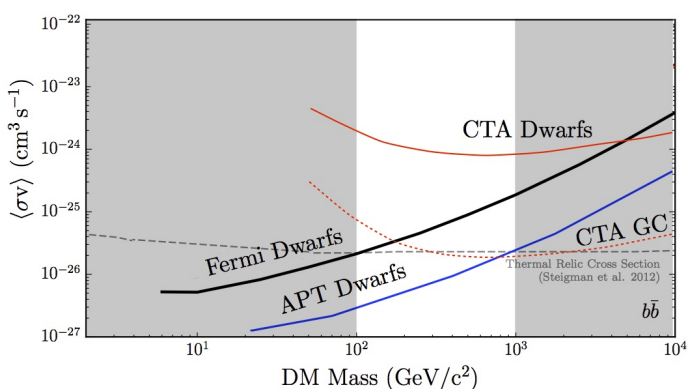

Figure 2: Estimated sensitivity of APT to DM. in a position to detect or rule out the entire natural parameter space for a thermal WIMP up to TeV mass scales where CTA measurements of the Galactic Center become the most sensitive search strategy (see Fig. 2).

The recent discovery of gravitational waves $(\mathrm{GWs})$ from a neutron-star merger by the LIGO collaboration [5] points to the potential impact of all-sky instruments for multi-messenger astronomy. These $n$-star mergers, like short $\gamma$-ray burst (GRB) sources, are difficult to study given the lag in re-pointing narrow-field instruments. Instantaneous all-sky coverage is key to detection and localization of these events; the $\gamma$-ray band is one of the few wavebands (over the entire electromagnetic spectrum) in which such coverage is possible. The very large effective area of APT in the $\mathrm{MeV}$ to multi-GeV regime would be ideal for detect-

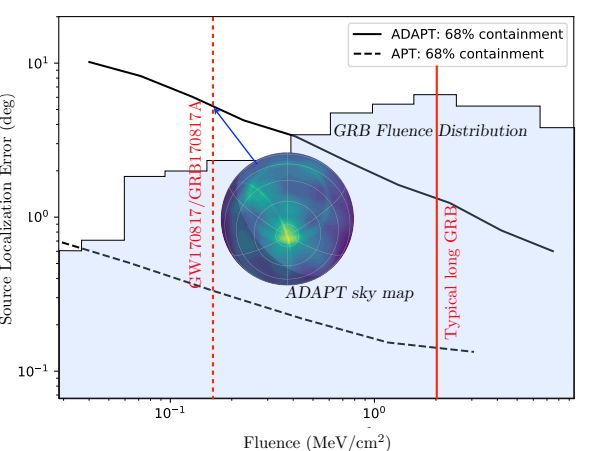

Figure 3: ADAPT and APT Compton event localization error versus fluence. Inset shows a simulated ADAPT event. ing emission from short GRBs and GW sources. For GRBs, the Fermi LAT has only detected the brightest events near the high end of the fluence distribution; hence, an improvement in effective area is as important as the larger instantaneous FoV.

Fig. 3 shows the sensitivity to GRBs of varying fluences (measured in the $0.3-10 \mathrm{MeV}$ band) for a typical burst spectrum for APT and ADAPT. (We note that this result shows a more optimistic estimate for APT than in [2] by including CsI edge detectors in the APT detector model.) The shaded background shows a part of the GRB fluence distribution measured with the Fermi GBM. For a burst with fluence approximately equal to the n-star merger event GW170817/GRB170817A (red dashed line) point-source localization would provide a positional uncertainty of $\approx 5^{\circ}$ for ADAPT and $\approx 0.4^{\circ}$ for APT. For a typical long burst (solid red line) ADAPT could localize the burst to within $\approx 1.5^{\circ}$ and APT to within $\approx 0.15^{\circ}$.

APT can also provide CR measurements that complement the $\mathrm{MeV} \gamma$-ray detections of nstar mergers. For many years it has been thought that ultra-heavy $(Z>30)$ r-process nuclei were synthesized in core-collapse supernovae (CCSNe). However, in recent years there have been a number of papers suggesting that a large fraction of r-process nuclei are synthesized instead in binary neutron star mergers (BNSM) (see, e.g., [6]). Although CCSNe are 1000 times more frequent than 
BNSM in the Milky Way, the amount of r-process material ejected in a BNSM is much larger than for CCSNe, and BNSM could therefore account for the observed abundances of r-process nuclei. Measurements of r-process nuclear abundances in CRs (in particular the actinides Th, $\mathrm{U}, \mathrm{Pu}$, and $\mathrm{Cm}$ ) provide a unique method of testing whether CCSNe or BNSM are responsible for the heaviest r-process nuclei in the contemporary cosmic rays. For example, if the source of the actinide nuclei is CCSN, ${ }_{94} \mathrm{Pu}$ (half-life $81 \mathrm{Myr}$ ) and ${ }_{96} \mathrm{Cm}$ (half-life $16 \mathrm{Myr}$ ) should be an appreciable fraction of ${ }_{90} \mathrm{Th}$ abundance since they would not have decayed. On the other hand, if BNSMs with lifetimes $\sim 100$ Myr [7] are the source of these nuclei, then ${ }_{96} \mathrm{Cm}$ should be essentially completely decayed and ${ }_{94} \mathrm{Pu}$ should be partially decayed. Elemental abundances determined by APT through multiple energy loss $(d E / d x)$ versus total $E$ measurement could provide an increase in statistics on ultra-heavy abundances orders of magnitude above the sensitivity of current experiments.

\section{Description of the APT Instrument}

APT would have a cross-sectional area of $3 \mathrm{~m}$ $\times 3 \mathrm{~m}$ and a height of $2.5 \mathrm{~m}$, consisting of $20 x y$ tracker and imaging CsI calorimeter (ICC) layers (Fig. 1). Wavelength shifting (WLS) fibers ( $2 \mathrm{~mm}$ square) covering thin $(\sim 5 \mathrm{~mm})$ CsI:Na tiles are used to collect and shift the UV/blue emission from the CsI:Na and pipe the light to SiPMs (Fig. 4). The tracker would be composed of $1.5 \mathrm{~mm}$ round scintillating fibers with two interleaved layers for both the $x$ - and $y$-coordinate determination. Centroiding the light collected by the orthogonal WLS fibers bonded to either side of the CsI crystals provides the $x y$ coordinates of the interaction (Fig. 5). The instrument has a depth of 5.8 r.l. and would weigh approximately $7,200 \mathrm{~kg}$, allowing it

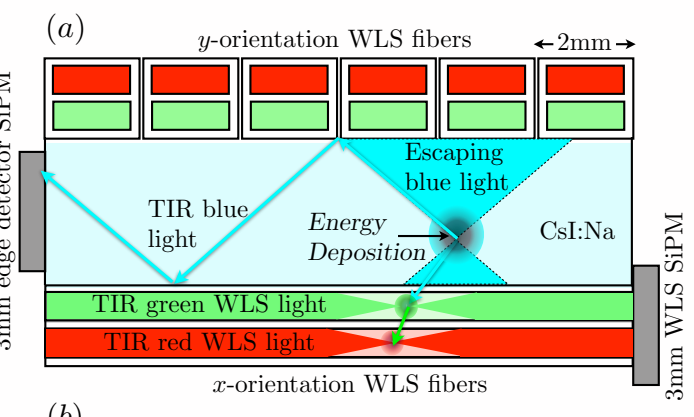

(b)

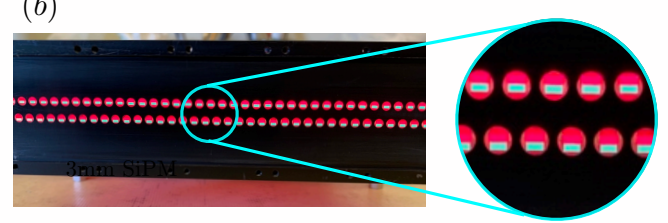

Figure 4: (a) Imaging CsI detector principle of to be lifted to a sun-Earth Lagrange orbit by a Falcon 9 heavy rocket. In such an orbit, Earth obscuration is minimized, and the up-down symmetry of the distributed calorimeter would allow the instrument to have twice the instantaneous FoV compared to that of the Fermi LAT.

The main detector volume is a passive, rigid structure composed of plastic fibers and CsI crystals supported by a carbon-fiber/foam structure. The long attenuation length of scintillating fibers allows the detector to be read out at the edges without embedded electronics or gaps in the detector volume. A total of 560,000 fiber/SiPM channels would be multiplexed into 80,000 waveform digitizer readout channels - significantly fewer than that of the Fermi LAT $(\sim 800,000)$.

The use of analog-pipeline waveform digitizers to read out the SiPMs is also key to the APT approach. The deep analog memory ( $\sim$ several $\mu \mathrm{sec})$ and double-buffered, self-triggered operation would provide ample trigger latency to process the slow CsI:Na signals to form an instrument trigger. FPGAs on the digitizer boards would be used for front-end data processing including hit detection, pulse integration, and coordinate and time centroiding. This allows a dramatic reduction in data rates and permits subsequent high-level data processing on flight computers. At the same time, the high-speed waveform digitization (100-250 Msps) provides a flexible readout that can characterize 
the fast plastic scintillator pulses, count photons in the weak CsI:Na signals for Compton scatters, and allow for reconstruction of saturated signals from high- $Z$ particles [1].

The new detector element for APT is the Imaging CsI Calorimeter (ICC) (Fig. 4). ह5: 80 This replaces the passive converter layers for a pair telescope and provides imaging calorimetry for high-energy events. Moreover, the crossed WLS fiber readout allows one to simultaneously measure the $x$ - and $y$-coordinates of a Compton scatter.

Sodium-doped CsI (CsI:Na) has a very high yield and a scintillation spectrum peak-

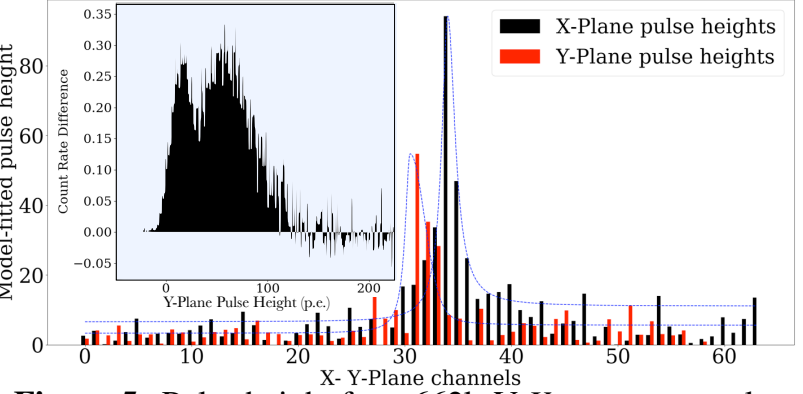

Figure 5: Pulse height for a $662 \mathrm{keV} X$-ray vs. $x, y$-plane fiber number. Inset: Cs-137 pulse-height distribution. ing at $\sim 420 \mathrm{~nm}$, well matched to common blue-sensitive photodetectors and green wavelengthshifting dyes. Fibers using green K-27 WLS dye efficiently absorb 420nm CsI:Na scintillation light and re-emit at $\sim 500 \mathrm{~nm}$. By using crossed $2 \mathrm{~mm}$ fiber planes (one in the $x$-direction on top and one in the $y$-direction on the bottom of the crystals) one achieves sufficient light collection to provide $x-y$ reconstruction (Fig. 5). The acceptance cone corresponding to the total internal reflection angle subtends a number of fibers, allowing one to centroid the interaction point in the CsI (Fig. 4). The light transmitted from the CsI into the core of each $2 \mathrm{~mm}$ WLS fiber produces secondary isotropic emission from the WLS dye; about $4 \%$ of this light is totally internally reflected to the end of the fiber to the SiPMs. Adding a second red layer can reclaim the green WLS light that escapes from the green WLS fiber, in principal nearly doubling the light reaching the SiPMs. We currently measure $\sim 180$ p.e. in response to Cs-137 $662 \mathrm{keV}$ X-rays (Fig. 5).

\section{ADAPT}

The ADAPT instrument will provide a demonstration of the full complement of detectors and readout electronics for APT. The mission benefits from the recent development of very-high-altitude, 60 million cubic-feet (Big60) balloons; above $\sim 100 \mathrm{keV}$, the atmospheric transmission at float improves from $60 \%$ (for a $39 \mathrm{MCF}$ balloon) to $85 \%$ (for a $60 \mathrm{MCF}$ balloon). Pointing knowledge will be obtained by a differential GPS system and a star camera, and results of realtime localization of GRBs would be relayed to the ground

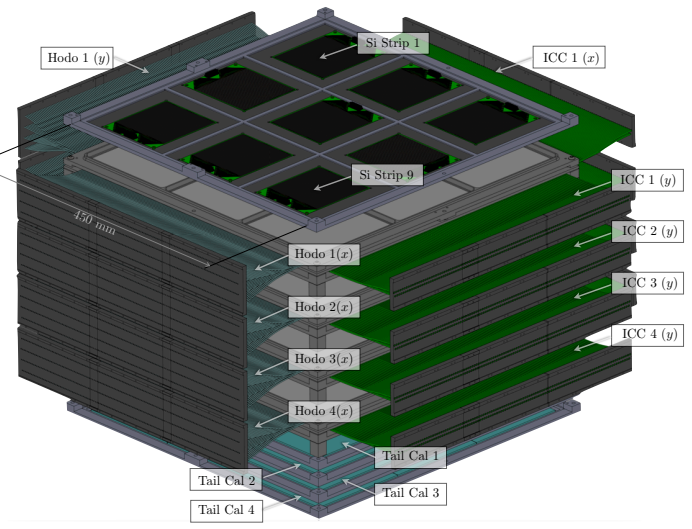

Figure 6: The ADAPT detector stackup. for multi-wavelength follow ups. The ADAPT detector will consist or four layers of ICC and scintillating-fiber tracker Hodoscope layers, and 4 layers of CsI tail counters (Fig. 6). A single layer of Silicon Strip Detectors (SSDs) on top provide a tagged calibrated beam through high-resolution charge measurements from protons to Fe, as for our CERN heavy-ion beam test [1]. Including this detector will also allow us to evaluate the potential for including a single SSD detector layer in the APT mission to enhance Compton reconstruction for low-energy events while also improving $\mathrm{CR}$ charge measurements. The tail counters are simple CsI layers that extend the depth of the calorimeter to 2.2 r.l. to provide energy measurements up to a few $\mathrm{GeV}$ [2]. 
The SSD plane consists of $9450 \mu \mathrm{m}$-thick Micron Si-strip detectors, each with active area $96 \mathrm{~mm} \times 96 \mathrm{~mm}$, covering about $40 \%$ of the $450 \mathrm{~mm} \times 450 \mathrm{~mm}$ cross section. The single-sided DCcoupled detectors with $641.5 \mathrm{~mm}$ pitch strips are nearly identical to those used in the CERN beam test [8]). For the strip readout, we will use the NRL1 or NRL2 ASIC to provide the low threshold readout of the strips. The NRL ASICs contains 32 channels with independent low-noise charge pre-amplifier, an analog shaper, stabilized baseline, a software trimmable discriminator, and a peak-and-hold circuit that functions as an analog memory of the peak amplitude on that channel. The NRL1 has a signal range of $2 \mathrm{~V}$ and four gain settings, 9.2-73.6 mV/fC, that correspond to 605 to $4840 \mathrm{keV}$ maximum range in Si. The measured resolution of the NRL2 with $30 \mathrm{pF}$ input capacitance is $2.2 \mathrm{keV} \mathrm{FWHM}$ at $90 \mathrm{keV}$ equivalent in Si [9]. The Ohmic side readout makes use of time-tested Amptek A250 preamplifiers and SuperTIGER pulse-height analyzer electronics to extend the dynamic range to signals from high- $Z$ CR nuclei.

The core detector system for ADAPT is the stack of four ICC/tracker modules with a $450 \mathrm{~mm} \times$ $450 \mathrm{~mm}$ cross-sectional area. Each ADAPT detector module consists of $9150 \mathrm{~mm} \times 150 \mathrm{~mm} 5 \mathrm{~mm}$ thick polished CsI:Na tiles. Tiles are bonded together at their edges using an optical silicone, and then bonded to two layers of $2 \mathrm{~mm}$ WLS fibers, 225 on each side, forming the WLS $x$ - and $y$-plane of the ADAPT instrument. This assembly is connected to the $\mathrm{Al}$ frame and carbon fiber substrates to support the CsI tiles. The $2 \mathrm{~mm}$ fibers are terminated in a machined Delrin block to align with the $3 \mathrm{~mm}$ SiPM carrier boards (Fig. 4). Staggering the layers allows the use of $3 \mathrm{~mm}$ SiPMs so that the WLS fibers under-fill the SiPM active area, while accommodating the $2 \mathrm{~mm}$ fiber pitch.

Each ADAPT tracker plane consists of two interleaved arrays of $3001.5 \mathrm{~mm}$ diameter round St. Gobain fibers. The 600 fibers in each $x$ or $y$-plane are assembled by first gluing one array of fibers into a machined grooved plate, then gluing the second array of fibers, offset by a fiber radius, to fill the interstitial positions of the first layer. The $1.5 \mathrm{~mm}$ fibers at the end of each plane are formatted into 4 rows of holes in Delrin end-plates,

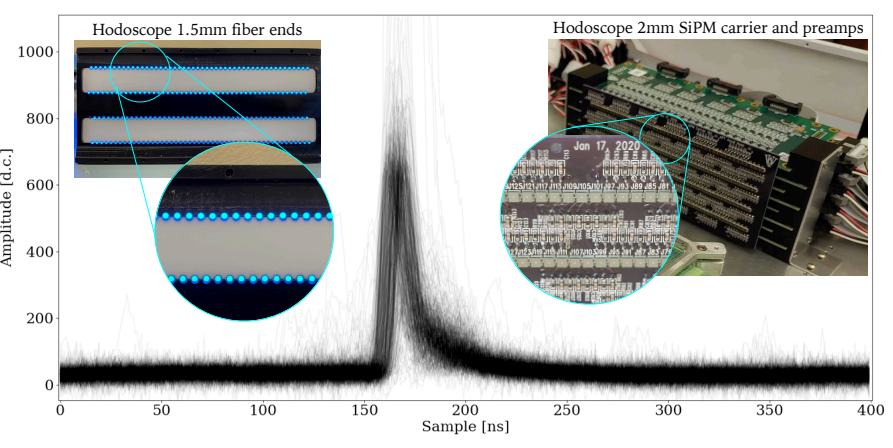

Figure 7: Muon pulses in single 1.5mm hodoscope fiber. Inset: fiber Delrin block, $2 \mathrm{~mm}$ SiPM carrier board and preamps. glued and polished by diamond milling. The Delrin block aligns the $1.5 \mathrm{~mm}$ fibers with a carrier board that holds four rows of $2 \mathrm{~mm}$ SiPMs (Fig. 7). Two crossed planes are bonded to form a single tracker layer and connected to the ICC detector plane. The SiPM carrier boards have highdensity connectors that connect to 3:1 multiplexing (mux) boards to a bank of preamplifiers and programmable SiPM bias voltage boards. The mux board spans the entire $450 \mathrm{~mm}$ length of the detector and combines corresponding fibers from the three $150 \mathrm{~mm}$ carrier boards, combining three fiber/SiPM channels into a single electronics channel. Preliminary measurements of the prototype show a light yield of $\sim 30$ p.e./MIP in a single fiber (see Fig. 7).

In addition to the fiber readout, the $x$ and $y$ edges of the CsI tiles are directly coupled to close-packed arrays of $3 \mathrm{~mm}$ SiPMs forming the edge detectors. These detectors collect the totallyinternally-reflected light in the CsI tiles that is not coupled into the fibers. Each edge detector includes a dual-range preamplifier that passively combines SiPM signals spanning a 150mm length. 
Three edge detectors cover the $x$ - or $y$-side of a module and are fed to 12 digitizer channels.

As for APTlite, the beam-test electronics for the ICC and tracker make use of discrete preamplifiers, individually programmable high voltage DACs for the SiPM bias, and TARGET C digitizer boards (utilizing the TC 16-channel 1Gsps analog pipeline and CT5TEA ASICs developed for the CTA project). For the ADAPT flight system, we will use front-end multiplexers, new SMART ASIC preamplifier/bias voltage boards, and low-power ALPHA analog-pipeline ASICs. The SMART (SiPM Multichannel ASIC for high-Resolution Cherenkov Telescopes) ASIC includes preamplifiers for $16 \mathrm{SiPM}$ inputs, each composed of a transimpedance amplification stage followed by a tail suppression circuit with programmable gain and pole-zero compensation. Preliminary measurements of the SMART ASIC coupled to a Hamamatsu $2 \times 2 \mathrm{~mm}^{2}$ SiPM (S13360-2050VE) showed that the preamplified signals can reach an amplitude up to $20 \mathrm{mV} /$ p.e. with a tail characterized by a $12 \mathrm{~ns}$ recovery time. The power consumption of the present ASIC design is $\sim 20 \mathrm{~mW} / \mathrm{channel}$, but R\&D is ongoing at INFN to reduce power consumption down to a few $\mathrm{mW} / \mathrm{channel}$.

While the performance of the TARGET C ASIC [10] is adequate for ADAPT, both its power requirements and its demands on I/O resources of the data collection/processing FPGA make it ill-suited for the space-based APT experiment. The ALPHA (Advanced Low-Power Hybrid Acquisition) ASIC is a much lower-power analog pipeline design developed tailored to APT. To scale to APT, the ALPHA employs self-triggering initiated by the companion CT5TEA ASIC (already sufficiently low power) and a daisy-chained serial readout to increase the number of channels processed per FPGA. The lower sampling rate (100-250 MHz) and limited size of the sampling array (256 cells) reduce power requirements. By using a double-buffering architecture, deadtime is reduced for self-triggering operation. For the full APT instrument with the nominal daisy-chain of twelve ALPHA ASICs (192 channels) and 64 such chains per FPGA, a single FPGA can process on the order of $12 \mathrm{k}$ channels. The ADAPT instrument will use a slightly less ambitious system, with a single PCB including 12 ATA devices, 12 ALPHA ASICs, and a single readout FPGA. Initial simulation results for the full APT configuration indicate that a few $\mathrm{mW} /$ channel is achievable, meeting the requirements for the total power budget of the full APT instrument. The design of the ASICs is nearly complete, and prototype fabrication is planned for fall of 2021.

For APT, we will use FPGA-based hit detection and centroid reconstruction similar to the design of a cross-strip readout demonstrated by our group [11]. After harvesting hit data, the most challenging aspect of the in-flight analysis is the requirement of a low-latency, high-throughput algorithm for prompt localization of GRBs through multiple Compton reconstruction. Demonstration of an algorithm that can meet timing and localization constraints is included in the contribution [3].

Detailed simulations were performed to evaluate the performance characteristics of ADAPT and APT [2] demonstrating that the APT design can achieve well over an order-of-magnitude larger geometry factor and effective area compared with Fermi. While the APT calorimeter is relatively shallow, fitting to the shower profile allows energy reconstruction to $\mathrm{TeV}$ energies. Angular resolution is similar to that of Fermi, although a large subset of events can be reconstructed with better angular resolution than Fermi, and the much larger effective areas at high energies will allow significantly enhanced resolution for hard spectrum sources. The use of active converter layers significantly improves the effective area and energy resolution for lower-energy pair events; even ADAPT will have sensitivity and energy resolution superior to the Fermi LAT at energies of $<30$ $\mathrm{MeV}$. In the Compton regime, APT would provide sub-degree burst localization and polarization 
constraints for a typical GRB fluence [2]. With an estimated burst rate of 120 GBM bursts per year with fluence $>2 \mathrm{MeV} / \mathrm{cm}^{2}$, scaling by the relative FoV of ADAPT and GBM, we expect about 3 GRBs to be detected in a 36-day flight with $<1.5^{\circ}$ localization accuracy, allowing potential for prompt multi-messinger observations and discovery with our small pathfinder ADAPT instrument.

\section{Acknowledgements}

This work was made possible through support by NASA grant 80NSSC19K0625 and NASA APRA award 20-APRA20-0148. The collaboration also acknowledges generous ongoing support from the McDonnell Center for the Space Sciences and the Peggy and Steve Fossett Foundation.

\section{References}

[1] Z. Hughes and J. Buckley, Characterization of a prototype imaging calorimeter for the Advanced Particle-astrophysics Telescope from an Antarctic balloon fight and CERN beam test, in Proceedings of 37th International Cosmic Ray Conference - PoS(ICRC2021), vol. 395, p. 137, 2021, DOI.

[2] APT collaboration, The advanced particle-astrophysics telescope: Simulation of the instrument performance for gamma-ray detection, in Proceedings of 37th International Cosmic Ray Conference - PoS(ICRC2021), vol. 395, p. 590, 2021, DOI.

[3] M. Sudvarg, J. Buhler, J. Buckley, W. Chen, Z. Hughes, E. Ramey et al., A Fast GRB Source Localization Pipeline for the Advanced Particle-astrophysics Telescope, in Proceedings of 37th International Cosmic Ray Conference - PoS(ICRC2021), vol. 395, p. 588, 2021, DOI.

[4] M. Ackermann et al., Searching for dark matter annihilation from milky way dwarf spheroidal galaxies with six years of fermi large area telescope data, Physical Review Letters 115 (2015) 231301 [1503.02641].

[5] B.P. Abbott et al., Observation of Gravitational Waves from a Binary Black Hole Merger, Physical Review Letters 116 (2016) 061102 [1602 . 03837].

[6] S. Shibagaki, T. Kajino, G.J. Mathews, S. Chiba, S. Nishimura and G. Lorusso, Relative Contributions of the Weak, Main, and Fission-recycling r-process, Astrophysical Journal 816 (2016) 79 [1505. 02257].

[7] O. Just, A. Bauswein, R. Ardevol Pulpillo, S. Goriely and H.-T. Janka, Comprehensive nucleosynthesis analysis for ejecta of compact binary mergers, MNRAS 448 (2015) 541 [1406.2687].

[8] J.F. Krizmanic, Y. Akaike, D.L. Braun, R.G. Bose, J. Buckley, G. DeNolfo et al., HNX/SuperTIGER Silicon Strip Detector Response to Nuclei in Lead Primary and Fragmented Test Beams, in 36th ICRC (ICRC2019), vol. 36, p. 94, July, 2019.

[9] E.A. Wulf, W. Hou, G. De Geronimo, J.M. Roberts, S.E. Boggs and B.F. Phlips, Front-end ASIC for germanium strip detectors, Nuclear Instruments and Methods in Physics Research A 954 (2020) 161230.

[10] J. Zorn et al., Characterisation and testing of CHEC-M-A camera prototype for the small-sized telescopes of the Cherenkov telescope array, Nucl. Instrum. Meth. A 904 (2018) 44 [1806.11308].

[11] J. Hyde, Data processing electronics for an ultra-fast single-photon counting camera, Master's thesis, Washington University Dept. of Electrical and Systems Engineering, St. Louis, MO, 8, 2020. 


\section{Full Authors List: APT Collaboration}

S. Alnussirat ${ }^{1}$, C. Altomare ${ }^{2}$, R. G. Bose ${ }^{3}$, D. Braun ${ }^{3}$, J. H. Buckley ${ }^{3}$, J. D. Buhler ${ }^{4}$, E. Burns ${ }^{1}$, R. D. Chamberlain ${ }^{4}$, W. Chen ${ }^{5}$, M. L. Cherry ${ }^{1}$, L. Di Venere ${ }^{6,2}$, J. Dumonthier ${ }^{7}$, M. Errando ${ }^{3}$, S. Funk ${ }^{8}$, F. Giordano ${ }^{6,2}$, J. Hoffman ${ }^{3}$, Z. Hughes $^{3}$, D. J. Huth ${ }^{3}$, P. L. Kelly ${ }^{5}$, J. F. Krizmanic ${ }^{9}, 10,11$, M. Kuwahara ${ }^{12}$, F. Licciulli ${ }^{2}$, G. Liu ${ }^{13}$, M. N. Mazziotta ${ }^{2}$, J. G. Mitchell ${ }^{14,15}$, J. W. Mitchell ${ }^{9}$, G. A. de Nolfo ${ }^{15}$, R. Paoletti ${ }^{16}$, R. Pillera ${ }^{17,2}$, B. F. Rauch ${ }^{3}$, D. Serini ${ }^{2}$, G. Simburger ${ }^{3}$, M. Sudvarg ${ }^{4}$, G. Suarez ${ }^{7}$, T. Tatoli ${ }^{15,18}$, G. S. Varner ${ }^{12}$, E. Wulf ${ }^{19}$, A. Zink ${ }^{8}$, W. V. Zober ${ }^{3}$

${ }^{1}$ Department of Physics and Astronomy, Louisiana State University, Baton Rouge, Louisiana 70803, USA, ${ }^{2}$ Istituto Nazionale di Fisica Nucleare, Sezione di Bari, I-70126 Bari, Italy, ${ }^{3}$ Department of Physics and McDonnell Center for the Space Sciences, Washington University, St. Louis, MO 63130, USA, ${ }^{4}$ Department of Computer Science \& Engineering, Washington University, St. Louis, MO 63130, USA, ${ }^{5}$ Department of Physics and Astronomy, University of Minnesota, Minneapolis, MN 55455, USA, ${ }^{6}$ Dipartimento di Fisica "M. Merlin" dell’Università e del Politecnico di Bari, I-70126 Bari, Italy, ${ }^{7}$ NASA Goddard Space Flight Center, Greenbelt, MD 20771, USA, ${ }^{8}$ Friedrich-Alexander-Universität Erlangen-Nürnberg, Erlangen Centre for Astroparticle Physics, D-91058 Erlangen, Germany, ${ }^{9}$ Center for Space Sciences and Technology, University of Maryland Baltimore County, Baltimore, Maryland 21250, USA, ${ }^{10}$ Astroparticle Physics Laboratory, NASA/GSFC, Greenbelt, Maryland 20771, USA, ${ }^{11}$ Center for Research and Exploration in Space Sciences and Technology, NASA/GSFC, Greenbelt, Maryland 20771, USA, ${ }^{12}$ Department of Engineering, University of Hawai $i \mathrm{i}$ at Mānoa, Honolulu, HI 96822, USA, ${ }^{13}$ Department of Physics and Astronomy, University of Hawai 'i at Mānoa, Honolulu, HI 96822, USA, ${ }^{14}$ Department of Physics, The George Washington University, Washington, DC 20052, USA, ${ }^{15}$ Heliospheric Physics Laboratory, NASA/GSFC, Greenbelt, MD 20771, USA, ${ }^{16}$ Università di Siena and INFN Pisa, I-53100 Siena, Italy, ${ }^{17}$ Politecnico di Bari, Department of Mechanics, Mathematics and Management, via Orabona, 4, I-70125 Bari, Italy, ${ }^{18}$ Department of Physics, Catholic University of America, Washington, DC 20064, USA, ${ }^{19}$ Naval Research Laboratory, Washington, DC 20375, USA 